\title{
Médiévales
}

Langues, Textes, Histoire

51 | automne 2006

L'Occident sur ses marges ( $\left.\mathrm{VI} \mathrm{e}^{\mathrm{e}} \mathrm{X}\right|^{\mathrm{e}}$ siècles)

\section{Anna Caiozzo, Images du ciel d'Orient au Moyen Âge. Une histoire du zodiaque et de ses représentations dans les manuscrits du Proche-Orient musulman}

Paris, Presses de l'Université de Paris-Sorbonne, 2003, 485 p., annexes, bibl., index (noms, thèmes).

\section{Nicolas Weill-Parot}

\section{(2) OpenEdition}

\section{Journals}

Édition électronique

URL : https://journals.openedition.org/medievales/1443

DOI : 10.4000/medievales. 1443

ISSN : 1777-5892

Éditeur

Presses universitaires de Vincennes

Édition imprimée

Date de publication : 1 décembre 2006

Pagination : 168-170

ISBN : 978-2-84292-193-4

ISSN : 0751-2708

Référence électronique

Nicolas Weill-Parot, «Anna Caiozzo, Images du ciel d'Orient au Moyen Âge. Une histoire du zodiaque et de ses représentations dans les manuscrits du Proche-Orient musulman », Médiévales [En ligne], 51 | automne 2006, mis en ligne le 12 mars 2007, consulté le 23 avril 2022. URL : http://

journals.openedition.org/medievales/1443; DOI : https://doi.org/10.4000/medievales.1443

Ce document a été généré automatiquement le 23 avril 2022

Tous droits réservés 


\section{Anna Caiozzo, Images du ciel d'Orient au Moyen Âge. Une histoire du zodiaque et de ses représentations dans les manuscrits du Proche-Orient musulman}

Paris, Presses de l'Université de Paris-Sorbonne, 2003, 485 p., annexes, bibl., index (noms, thèmes).

Nicolas Weill-Parot

1 Depuis les travaux pionniers d'Aby Warburg, Franz Boll, Fritz Saxl et Wilhelm Gundel, les historiens de l'art ont insisté sur l'intérêt majeur des représentations des figures célestes. Il manquait un ouvrage d'ensemble sur les traditions iconographiques de ces représentations pour le monde islamique. Cette tâche monumentale et extrêmement ardue, tant les sources sont hétérogènes et d'une inégale richesse suivant les périodes et les supports, Anna Caiozzo, à la fois historienne de l'Islam «médiéval» et historienne d'art, s'en est acquittée dans un imposant ouvrage.

2 L'un des fils directeurs de cette étude tient dans la distinction entre plusieurs zodiaques. Le zodiaque est une bande de ciel de part et d'autres de l'écliptique (trajectoire apparente du soleil dans sa révolution annuelle). Dans cette bande sont aussi comprises les trajectoires des six autres astres mobiles. Anna Caiozzo part d'une distinction fondamentale entre le «zodiaque sidéral des constellations» et le "zodiaque tropique des signes». Le premier correspond à douze des quarante-huit constellations d'étoiles fixes perçues à travers la bande zodiacale. Le second zodiaque, tropique, est défini par les douze parties égales qui balisent la course annuelle du soleil de l'équinoxe de printemps (lorsque la trajectoire apparente du soleil coupe l'équateur céleste au point vernal). Au zodiaque des astronomes (zodiaque sidéral) lié à l'observation du ciel, et à celui des astrologues (zodiaque tropique) lié à la prédiction des événements ici-bas, A. Caiozzo en ajoute un troisième, celui des magiciens (dérivé du deuxième). Cette triple distinction forme l'armature de son étude. 
Une première partie est consacrée au "ciel de l'astronome». A. Caiozzo retrace l'histoire du zodiaque de l'Antiquité mésopotamienne à l'Islam. Elle remarque que les mondes gréco-romain et préislamique ne distinguent pas dans les modes de représentation entre les zodiaques sidéral et tropique, malgré la connaissance du décalage qui existe entre eux, connaissance acquise depuis la découverte de la précession des équinoxes par Hipparque (II ${ }^{\mathrm{e}}$ siècle). L'iconographie du zodiaque paraît se fixer avec les Aratea, c'est-à-dire les ouvrages se situant dans la lignée d'Aratos de Soles. Et c'est bien ce modèle que l'on retrouve dans le tout premier zodiaque connu de la période islamique, celui de la coupole du caldarium du palais ummayade de Qusayr 'Amra en Jordanie ( $\mathrm{vIII}^{\mathrm{e}}$ siècle). Cependant au siècle suivant les astronomes arabopersans proposent une nouvelle représentation $d u$ zodiaque; et la tradition du zodiaque astronomique en terre d'Islam repose en grande partie sur l'œuvre d'al-Sufî (903-986 ap. J.-C.) et son Livre des Étoiles Fixes, ouvrage illustré suivant ses propres indications, qui s'inscrit dans un travail de révision de l'Almageste de Ptolémée. En étudiant plusieurs manuscrits de cet ouvrage du XI ${ }^{\mathrm{e}}$ au XvII ${ }^{\mathrm{e}}$ siècle, A. Caiozzo conclut que, tout en conservant pour une bonne part les modèles antiques, hellénistiques et romains, les astronomes arabes ou persans ont introduit un certain nombre de modifications, lesquelles témoignent à la fois d'un souci d'exactitude astronomique et d'une méconnaissance de la mythologie. Les signes humains de ce zodiaque, en particulier, sont orientalisés. La tradition iconographique d'al-Sufî a été connue de l'Occident, notamment grâce aux traductions de la cour d'Alphonse X de Castille (milieu du XIII ${ }^{e}$ siècle), mais cette transmission fut sans lendemain. A. Caiozzo suit ensuite la postérité en terre d'Islam de cette tradition dans des ouvrages non astronomiques et dans d'autres supports (globes célestes) dont les commanditaires furent des califes, des princes ou d'autres « amateurs éclairés ».

4 Les deuxième et troisième parties sont consacrées au «ciel des astrologues »: les représentations astrologiques des planètes et du zodiaque. Le "père» de l'astrologie arabo-persanes est, sans conteste, l'astrologue Abû Ma'shar ( $x^{e}$ siècle). Après avoir présenté la discipline astrologique, A. Caiazzo étudie les supports les plus divers: miroirs, métaux (monnaies, vases) ou manuscrits (en particulier le ms. BnF Pers. 174, XIII ${ }^{\mathrm{e}}$ siècle, du Daqấ'iq al-haqấ'iq). La tradition iconographique des planètes remonterait aux $\mathrm{IX}^{\mathrm{e}}-\mathrm{x}^{\mathrm{e}}$ siècles et tirerait ses caractéristiques de diverses influences: dieux mésopotamiens, fonctions astrologiques hellénistiques, hermétisme et traditions indiennes. L'auteur attribue un rôle crucial dans ce creuset à l'énigmatique et célèbre secte des Sabéens de Harrân, demeurée païenne et astrolâtre en terre d'Islam au moins jusqu'au $\mathrm{XI}^{\mathrm{e}}$ siècle. A. Caiozzo apporte ainsi de nouveaux éléments à la recherche sur cette fascinante secte étudiée notamment par D.Chwolsohn, J. Hjärpe, Y. Marquet, M. Tardieu, D. Pingree et T. Green. La formation du zodiaque astrologique est plus lente et plus complexe. Un zodiaque astrologique ne vise pas à la description des constellations zodiacales, mais à la représentation symbolique d'un signe en association avec la planète dont il est le domicile. À la suite d'une démonstration très minutieuse (et parfois ardue pour le lecteur), A. Caiozzo met en évidence l'existence de deux types: un zodiaque où cette association est systématique et un autre dit "mixte " où cette association n'est pas généralisée; ainsi certains signes demeurent proches du modèle astronomique (Cancer, Scorpion, Poissons et Balance), d'autres sont altérés (Bélier, Capricorne), d'autres enfin ont "assimilé » dans leur représentation la planète à laquelle ils sont astrologiquement associés (Gémeaux, Vierge, Sagittaire et Verseau). 
Pour finir, A. Caiozzo aborde le "ciel du magicien et du sorcier ", c'est-à-dire les représentations à but talismanique: qu'ils s'agissent de talismans des signes du zodiaque ou des planètes ou de ceux des vingt-huit mansions lunaires auxquelles sont associés des jinns et des anges. Elle donne ainsi des clefs pour comprendre certaines des sources essentielles de la magie astrale (comme le Ghâyat-al-Hakim, traduit en latin sous le nom de Picatrix).

L'ouvrage d'Anna Caiozzo, muni d'un index (auteurs, notions) est extrêmement riche : au-delà du fil directeur consistant à retracer des traditions iconographiques jusque-là fort mal connues pour l'Orient islamique, c'est bien aussi une histoire de l'astrologie (ses principes, ses utilisateurs, sa signification culturelle et sociale) qui se lit en filigrane à travers ces pages denses, argumentées et illustrées de nombreuses figures. Un épais dossier d'annexes (tableaux descriptifs des diverses figures célestes et de belles planches en couleur) font de cet ouvrage un outil indispensable pour les recherches sur l'iconographie astrologique non seulement dans le monde islamique, mais aussi par ricochet dans l'Occident médiéval. 